\title{
Embedded RFID and Wireless Mesh Sensor Network Materializing Automated Production Line Monitoring
}

\author{
C.Z. Zulkifli ${ }^{a, *}$, H.N. HASsAN ${ }^{a}$ AND W. Ismail ${ }^{b}$ AND S.N. SEMUnAB ${ }^{d}$ \\ ${ }^{a}$ Sultan Idris Education University, Computing Department, Tanjung Malim Perak, Malaysia \\ ${ }^{b}$ Universiti Sains Malaysia, Electrical and Electronic Engineering, Penang, Malaysia \\ ${ }^{c}$ Faculty of Art, Computing and Creative Industry Universiti Pendidikan Sultan Idris, \\ Tanjung Malim, Perak, Malaysia \\ ${ }^{d}$ Sultan Idris Education University, Computing Department, Tanjung Malim, Perak, Malaysia
}

\begin{abstract}
In industrial manufacturing, wireless network can be used in supply chain, retail stock management, electronic security keys, and theft prevention. A proactive and cost effective solution advancement is needed in the industrial manufacturing to monitor and control output performance at a manufacturing plant. It is with this reason that the manufacturers require an efficient communication and real time feedback to maximize uptime and improve productivity. Consistent with the future market of new technologies deployment this invention proposes a new complete solution method and architecture of embedded active radio frequency identification with counter system through wireless mesh sensor network platform and smart data processing adopted through web-based monitoring system for industrial automation. The ability of the developed system to function in machine-to-machine wireless communication and the adoption of web-based monitoring and data centralization contributes improvement in reducing errors with human intervention. In this paper we discuss the framework approach of a smart web-based monitoring and data acquisition system on wireless mesh sensor network platform. The evaluations and measurements have been conducted in real world environments which are conducted at selected industrial production factory.
\end{abstract}

DOI: 10.12693/APhysPolA.128.B-86

PACS: 84.84.- $\mathrm{x}, 07.57 .-\mathrm{c}$

\section{Introduction}

Internet base monitoring and control system has been introduced in recent years in numerous applications. This technology contributes a lot of benefits for many areas not only in the industry but also in the field of military education and hospitality. Now a day's famous research work on the Internet based process control has resulted in small-scale demonstrations like Sun Microsystems and Cyberonix Foxboro and Valmet. Most of them were developed in Java. Additionally the Open Process Control (OPC) Foundation is working on supporting XML within Visual Studio so that the Internet based process control using XML is widely used. Intuitive Technology Corporation has provided web@aGlance for feeding real-time data to a Java graphics console [1]. The International Federation of Control (IFAC) has held the first workshop on Internet Based Control Education in Spain in 2002 [1]. The SCADA system funded by the European Council targets Internet based protocols enables the monitoring and optimization of the process via the web [1]. The objectives of establishing Internet based process control systems is to enhance rather than replace computer based process control systems by adding an extra Internet level in the hierarchy [2]. Even though SCADA system is the solution of controlling and managing in wide range of industries, the proposed project

*corresponding author; e-mail: chezalina@fskik.upsi.edu.my holds the promise of replacing the existing controlling system. In fact, the proposed system has the advantages where RFID module is embedded in it and it is designed specifically for manufacturing sector. Using technology is not enough without developing managerial communication and technical abilities. In web-based monitoring and data acquisition system (MCDAS) we designed the system that works best to help industrial sector to meet responsibilities.

Implementation of wireless mesh network for monitoring of production output achievement can revolutionize industrial processing and help industries meet the demands of increasing competitiveness [3]. Intelligent ZigBee technology with $24 \mathrm{GHz}$ active radio frequency identification (RFID) as wireless network and web-based monitoring in industrial environments enables real-time data sharing throughout a facility and this will increase industrial safety efficiency and productivity. This automated monitoring technology offers reliable autonomous and improved process control enhancing safety, ameliorating product quality, increasing yield, and reducing costs [4].

In order to make web-based monitoring system more reliable ZigBee is integrated with MCDAS in our proposed system to achieve real time automation monitoring and control in industrial manufacturing environment. Mesh networks consist of low-cost battery-powered sensor modules and embedded networking intelligence [5]. ZigBee is a type of LRWPAN technology and is built upon the lower layers of the IEEE 802154 LRWPAN standard [1]. Furthermore, ZigBee is also a growing technol- 
ogy that will gain more advantage in industrial automation [6]. Hence we applied ZigBee technology in this industrial automation.

This paper is structured as follows. Section 2 describes the system components and characteristics of MCDAS and wireless applications. Section 3 discusses result and discussion. Finally, Sect. 4 gives conclusion.

\section{System components}

This research aims to design and implement an application of wireless mesh network for real-time monitoring of production output achievement in an industrial context. To achieve this goal, the following objectives are considered. Most concerning is to perform evaluation in terms of performance and flexibility relative to environment threats to wireless communication technology for industrial applications. Then main characteristics of wireless sensor networks used in industrial automation applications are investigated including network topology and architectures, real-time restrictions, reliability, power consumption, and life-time issues [7]. This research incorporates IEEE 02.15.4/ZigBee, communication protocols used with wireless sensor networks and dedicated solutions to include ZigBee technology in $2.45 \mathrm{GHz}$ active RFID to support wireless mesh network in order to develop a fully automated and embedded system for monitoring and data acquisition system in industrial application. A thorough investigation on how to perform the hardware integration with the webbased system is also the main part of this research. Apart from concern on hardware and communication side, this study also focuses on software development beyond the ordinary programming language. An ordinary monitoring system is not web-based, whereas the proposed MCDAS in this research uses web-based programming language. Web-based programming and architecture system are introduced in this study to design and develop a fully automated and embedded system for real time monitoring and data acquisition system in industrial application. Web-based programming technology is a challenging technology in order to make this automation more efficient and reliable [8].

Our proposed system is divided into three main portions. First stage of this system consists of integration of collecting data. In second stage output from first part will be transmitted by active RFID tag to RFID reader using ZigBee wireless technology. In this part it is necessary for the RFID reader to communicate to the real time database server. The last stage is web based monitoring part where the end user can access the system to view the outputs produced from each line through internet access. By integrating the sensor counter system and ZigBee technology in $2.45 \mathrm{GHz}$ active RFID to support wireless mesh network, the web-based monitoring system, i.e., MCDAS will be developed, which would be enable to update the details of production line activities.

Figure 1 shows the block diagram for development of proposed system. The hardware elements consist of sen- sors counters and active RFID tags. As the input to counter system, a photo sensor is integrated with the counter. At the end of each line the photosensor detects the products appearing in front of it. The system will send the product count from the sensor to the counter. Each counter system needs to be paired with their individual RFID tags. The circuit is designed to enable RFID tag to receive the power from microcontroller unit (MCU). One of the fundamentals obstacles in the RFID system is the power consumption [9]. Clearly with the circuit enhanced capabilities the problem of power in the RFID tag can be eliminated. This RFID tag will update the data to the reader accordingly when the data from counter is renewed. The RFID reader will receive the data from the active RFID tag through ZigBee network [10].

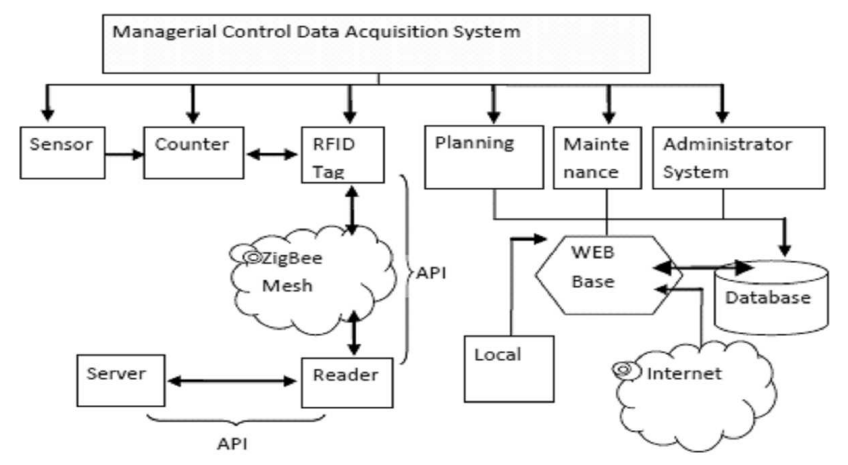

Fig. 1. Development of hardware and software system.

\section{Result and discussion}

In the experiments we explore the capabilities and benefits of MCDAS software system compared to traditional monitoring system in industrial environment applied for 9 line electronic goods production industry in Seberang Perai Penang, Malaysia. It has been proven that the invention and innovation introduced with the use of RFID as the main platform do contribute positively to research platform in specific and real world implementations in general as described. MCDAS report, on the other hand, provides automated, accurate collection of run time, cycle time, production and $10 \%$ of efficiency that ensure job cost to be accurate. The evaluation data shows that there is no more unaccounted for downtime because the information is automatically printed at the end of each shift. In the production report the time of input from the embedded hardware has been recorded. This means that the production line is running. If the production line is down, automatically the embedded hardware cannot send the data to the system. The design relationship between the data acquisition system and the overall system components provides the smart embedded system architecture to contribute the positive impact to the organization [11]. Capability of monitoring from out of factory provides lower downtime and higher monitoring efficiency. Figure 2 shows the downtime trend for two 


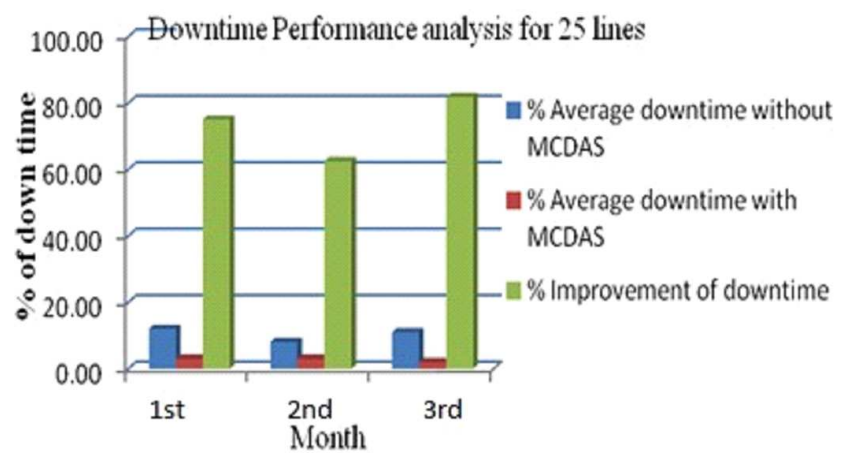

Fig. 2. Downtime analysis based on three months.

conditions which are with the proposed system deployment and with the existing traditional system. The green bar represents the improvement percentage of downtime. It records an $80 \%$ improvement with the implementation of the proposed system. These measurements are captured based on 3 months trial at the company factory in Penang, Malaysia.

The downtime of production lines is directly contributed to the company's productivity and can measure the managerial monitoring and control rate [12]. Decrease of downtime will cause the increase in productivity and yield [13]. The trend shows that the efficiency of managerial monitoring capability is increased with the implementation of MCDAS. Figure 3 shows a comparison result based on a three-month pilot test for four main constraints of the company measurement, which is the yield, productivity, downtime, and efficiency of monitoring system. All the main constraints show a positive improvement after MCDAS implementation. The downtime contributes an average of $80 \%$ improvement for three months of MCDAS implementation due to the increase of monitoring from the supervisor and management through MCDAS machine-to-machine (M2M) communications. A supervisor then has extra time which averages at four hours per shift to monitor the production line process, since no time has to be wasted on data collection, data entry and reports because MCDAS provides an automated data collection data entry system and real time reports. The result shows that with the synchronization of the yield and productivity and since the downtime is reduced the yield and productivity is increased, triggering the company to gain a higher profit.

Architecture of the software platform also contributes to the effectiveness and efficiency of the implementation. Table simplifies the comparison of a few systems renowned with the proposed system. The SCADA system is a widely known provider in providing the monitoring and data acqusition system. SCADA is more focussed on the gas and piping industry [14]. In addition to this, BARCO is famous for supplying system monitoring to the industries especially for the carpet manufacturing company. BARCO uses Bluetooth technology in a system designed for limited accessible communication distance of $15 \mathrm{~m} \mathrm{[14]}$.

TABLE

Comparison of system providers [15].

\begin{tabular}{c|c|c|c|c|c|c|c}
\hline \hline $\begin{array}{c}\text { System } \\
\text { Name }\end{array}$ & $\begin{array}{c}\text { Embedded } \\
\text { RFID } \\
\text { and WSN }\end{array}$ & $\begin{array}{c}\text { Web-based } \\
\text { system }\end{array}$ & $\begin{array}{c}\text { Low } \\
\text { cost }\end{array}$ & $\begin{array}{c}\text { Automated moni- } \\
\text { toring compared } \\
\text { standard time, } \\
\text { target and actual }\end{array}$ & $\begin{array}{c}\text { M2M wireless } \\
\text { communication }\end{array}$ & $\begin{array}{c}\text { Communication } \\
\text { range }\end{array}$ & $\begin{array}{c}\text { Flexible design, } \\
\text { adding \& modifi- } \\
\text { cation software } \\
\text { \& hardware }\end{array}$ \\
\hline SCADA & $\mathrm{X}$ & $\sqrt{ }$ & $\mathrm{X}$ & $\mathrm{X}$ & $\mathrm{X}$ & - & $\sqrt{ }$ \\
ABACUS & $\mathrm{X}$ & $\sqrt{ }$ & $\mathrm{X}$ & $\mathrm{X}$ & $\mathrm{X}$ & $\mathrm{X}$ \\
BARCO & $\mathrm{X}$ & $\mathrm{X}$ & $\mathrm{X}$ & $\mathrm{X}$ & $\mathrm{X}$ & $\begin{array}{c}15 \mathrm{~m} \\
\text { X0 to 200 m }\end{array}$ \\
MCDAS & $\sqrt{ }$ & $\sqrt{ }$ & $\sqrt{ }$ & $\sqrt{ }$ & $\sqrt{ }$ & $\begin{array}{c}\text { Indoor stand alone not yet } \\
\text { in mesh network }\end{array}$ & $\sqrt{ }$
\end{tabular}

On the whole, the proposed embedded MCDAS offers various flexibilities compared to others. The technology used by other work is not so extensive as the one conducted in the proposed system, such that the invention and innovations which have been adapted to the RFID technology provide positive significant results to be applied in real world environment such as the manufacturing company. Most of the previous work tend to stick to only one technology and does not include the invention and innovation of any other technology so that its capabilities can be optimized.

\section{Conclusion}

In this paper an overview of comparatively new technology and architecture for web-based system and active RFID integrated in wireless mesh network in industrial applications has been presented. The state of the art has been summarized by describing the framework of "real industrial environment" case study. Specifically wireless mesh network can be exploited to reduce cost and time constraints to gain optimum profit. The intent is to show ways on how industrial manufacturers can use wireless technologies to improve their monitoring sys- 


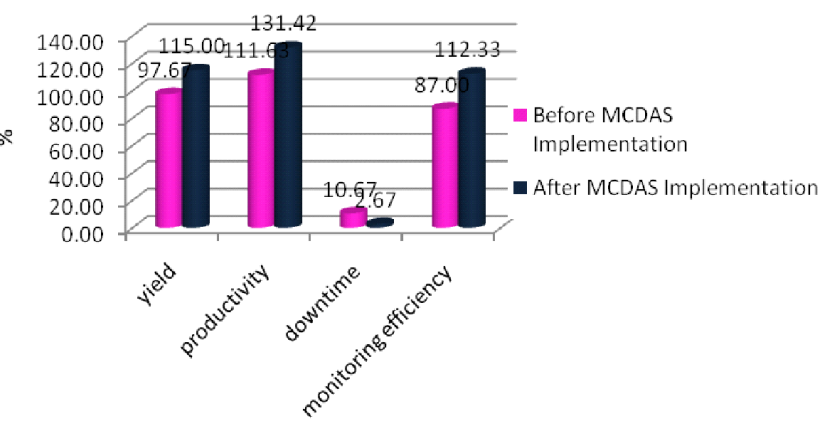

Fig. 3. Productivity performance of before and after MCDAS implementation for 3 months pilot test.

tems. The proposed designed and developed system has managed to materialize the M2M communication, so as to replace the human-to-machine (H2M) communication, and as such, has increased the efficiency rate of the interaction and communication process between most elements including the individuals, organization as well as the machines. On the whole, it can be concluded that the proposed new architecture design which merges four technologies into one solution is highly suitable and compatible and hence, fulfills the requirement of the production line output monitoring application.

\section{References}

[1] T. Handersan, D. Kotz, I. Abyzov, Comput. Networks J. 52, 2690 (2008).

[2] A. Flammini, P. Ferrari, D. Marioli, E. Sisinni, A. Taroni, Microelectron. J. 40, 1322 (2009).

[3] M. Chen, S. Gonzales, V. Leung, M. Li, Wireless Commun. IEEE 17, 37 (2010).

[4] D.L. Wu, W.W.Y. Ng, D.S. Yeung, H.L. Ding, in: Proc. Eight Int. Conf. on Machine Learning and Cybernetics, Vol. 4, IEEE 2009, p. 2330.
[5] H.A. Kadir, M.H.A. Wahab, S.N. Aqmariah, J. Social Sci. 5, 206 (2009).

[6] A. Mockus, R.T. Fielding, J. Herbsleb, ACM Trans. Software Eng. Methodol. 11, 309 (2002).

[7] I.F. Akyyildiz, X. Wang, W. Wang, Comput. Networks J. 38, 393 (2005).

[8] J.H. Khor, W. Ismail, M.I. Younis, M.K. Sulaiman, M.G. Rahman, J. Wireless Person. Commun. 59, 17 (2010).

[9] J. Crichigno, M.Y. Wu, W. Shu, Ad Hoc Networks J. 6, 1051 (2008).

[10] L. Wang, K.C. Tan, Modern Industrial Automation Software Design, Book Chapter Part 1, Institute of Electrical and Electronics Engineers Inc., 2006, p. 1.

[11] W. Zhao, A Study of Web-Based Application Architecture and Performance Measurements, School of Information Systems Curtin University, Perth 1999.

[12] S.W. Chang, A Case Study of a Web-Based Timetabling System, Book Series, Springer, Berlin 2001, p. 236.

[13] Q.H. Wu, D.P. Buse, J.Q. Feng, P. Sun, Int. J. Automat. Comput. 1, 17 (2004).

[14] M. Francheschinis, M.A. Spirito, R. Toamsi, G. Ossini, M. Pidala, in: Proc. Second Int. Conf. on Sensor Technologies and Application, 2008, p. 282.

[15] C.Z. Zulkifli, R. Abdulla, W. Ismail, M.G. Rahman, in: Int. Joint Conf. on Advances Processing and Information Technology, LNICST, Vol. 62, Springer, 2011, p. 7. 